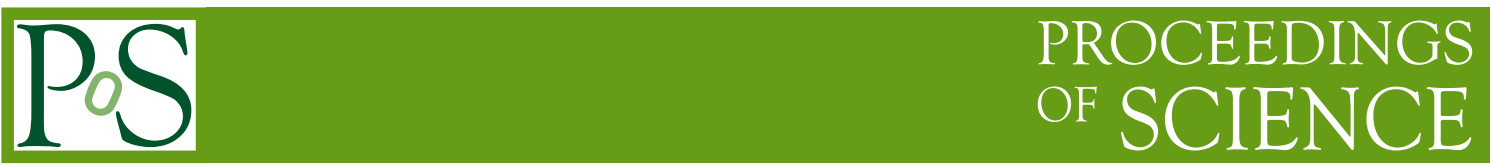

\title{
A JEM-X Catalog of X-ray Sources
}

\author{
Niels J. Westergaard* \\ National Space Institute, Technical University of Denmark \\ Juliane Maries Vej 30, Copenhagen, Denmark \\ E-mail: njw@space.dtu.dk
}

The JEM-X catalog of X-ray sources presented here results from running OSA8+ for all the public data up to and including revolution 728 . Sources detected in individual science windows are found down to a sensitivity limit of about $10 \mathrm{mCrab}(5-15 \mathrm{keV})$. This way shortlived sources may be detected. A search for weaker, persistent, sources has been done in deep mosaic images that have been produced with all available observations for a large number of sky regions. The two resulting catalogs hold 158 and 179 sources respectively, but the combined catalog consists of 209 sources. This catalog can be downloaded as a FITS binary table file with source information such as names, positions, and fluxes at the PoS web page for the conference.

The Extreme sky: Sampling the Universe above $10 \mathrm{keV}$ - extremesky2009,

October 13-17, 2009

Otranto (Lecce) Italy

${ }^{*}$ Speaker. 

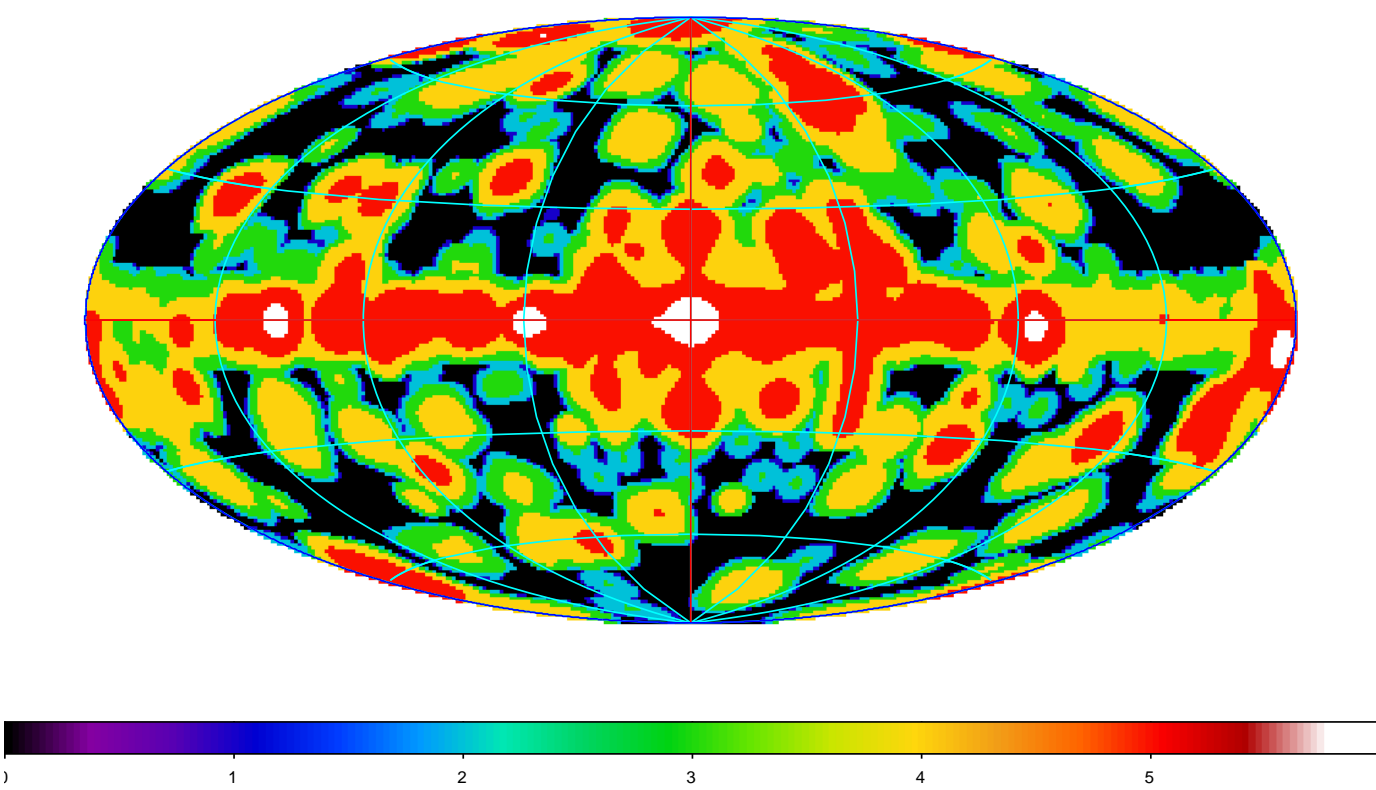

Figure 1: Map in galactic coordinates of exposure time in the science windows used for the present catalog. The color bar gives the logarithm (base 10) of the lower range of exposure time in seconds.

\section{Introduction}

JEM-X (Lund et al., 2003) is the X-ray monitor onboard the INTEGRAL satellite. Its role is to get a better position accuracy for the detected sources as well as an extension of the spectra to lower energies, i.e. below $20 \mathrm{keV}$. JEM-X has a narrower Field-Of-View than the main instruments, IBIS and SPI, the gamma-ray imager and spectrometer. For all three instruments imaging is obtained by a coded mask and in order to minimize systematic effects - in particular for SPI a so-called dithering is performed. It consists of offsetting the pointing direction in a fixed pattern around the targeted source. The side-effect of this is a wider sky coverage for JEM-X, but also less time on the target source.

This catalog summarizes the source detection by JEM-X in all the public data available until November 2009. The search for sources has been done in two ways: in individual pointings (Science Windows) and in mosaic images produced by combining all the images available in the given region.

Both JEM-X instruments have been used, JEM-X2 through INTEGRAL revolutions 39- 170 and JEM-X1 from 170 to 728 (with some exceptions due to e.g. Solar particle emission). A large part of the sky is covered as illustrated in Fig. 1 which is a map of the exposure time obtained by combining JEM-X1 and JEM-X2.

The most comprehensive INTEGRAL source catalog is the 4th IBIS/ISGRI soft gamma-ray survey catalog (Bird et al., 2010) that contains 723 sources. This number is greater than the number of source in the catalog presented here because the ISGRI FOV is much larger than the JEM-X FOV and the detector area is also larger. 


\section{Data analysis}

\subsection{Observational data}

All science windows i.e. individual pointings of typical duration between $1500 \mathrm{~s}$ and $2000 \mathrm{~s}$ have been analyzed for sources in the three standard energy intervals as defined in OSA8 (see next section): $3-7,7-11$, and $11-20 \mathrm{keV}$. All public JEM-X data have been used at the time of the analysis i.e. including revolutions $26-728$.

The three images produced (one for each energy band) are searched independently for peaks characterized by a detection significance which is the highest peak value divided by the RMS value of the immediate surrounding in the image. A source candidate is reported by the imaging software if it appears in at least two of the images with a detection significance larger than 3 or in a single image with a detection significance larger than 10. Its following inclusion in the catalog depends on either an identification or a visual (human) verification.

\subsection{Analysis software}

The analysis software system is OSA-8 (Offline Analysis Software) by ISDC (INTEGRAL Science Data Centre, Versoix, Switzerland). The main image production and analysis tool is 'j_ima_iros' that has gone through important improvements for the OSA8 version. The version used here includes a bug fix so that the source finding is improved at the image edges (will be delivered for OSA9). The flux extraction is also based on that tool.

Similarly the image source localization tool 'j_ima_src_locator' used for searches in the mosaic images and peaksize determination has been improved.

\subsection{Reference catalog}

The ISDC reference catalog (initiated by Ebisawa et al., 2003) version 30 had to be updated slightly in order not to let the few erroneous source positions and a single omission stand out in Fig. 2. The Crab position (which is given as the pulsar position) has been replaced with the centroide Crab Nebula position (Westergaard et al. 2007). The star 'Algol' has been added, since it has been detected with JEM-X.

\section{Source localization accuracy}

The error of the source localization has a counting statistics and a systematic component. The analysis of Budtz-Jørgensen et al. (2006) demonstrates a weak dependence on temperature of the satellite structure measured at the mask which has been taken into account in the present analysis. Fig. 2 shows the deviation between the source positions combined from all detections properly weighted i.e. by detection significance and the reference catalog positions as a function of the combined detection significance for the individual sources.

The $2 \sigma$ confidence limit for source localization in single science windows is defined as the radius that contains $95 \%$ of the individual detections is given in Fig. 3 together with the $1 \sigma(67 \%)$ result. 


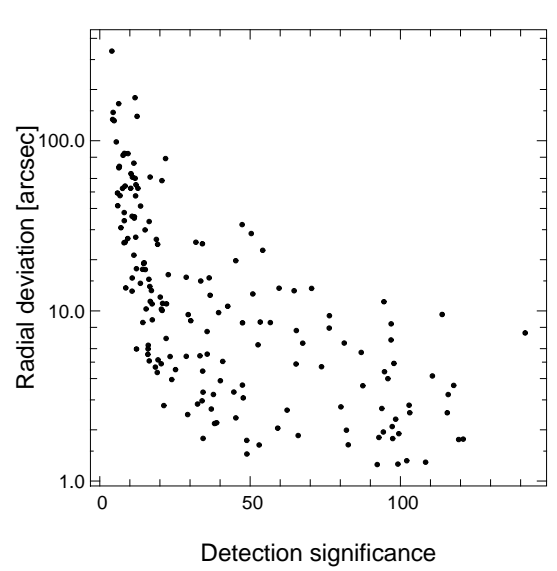

Figure 2: The source position deviation from the reference catalog plotted against the combined detection significance.

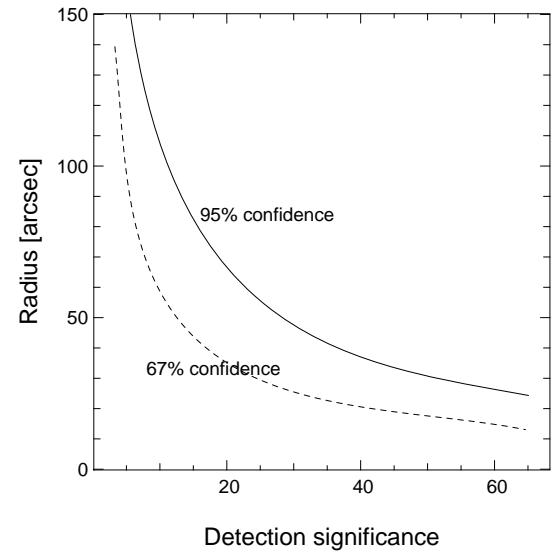

Figure 3: The 67 and 95\% confidence limits for the localization accuracy determined as a function of the detection significance.

Individual sources with large deviations between the JEM-X position and the catalog position have led to not only updates of the ISDC reference catalog but also to updated source positions in the SIMBAD catalog.

\section{Detection sensitivity}

The JEM-X sensitivity has been determined as the lower intensity limit of the identifiable sources. The exposure time was selected to be around $2000 \mathrm{~s}$, which is typical for the INTEGRAL science window durations. Fig. 5 shows the result given in cgs units in the interval where JEM-X is most sensitive (highest effective area).

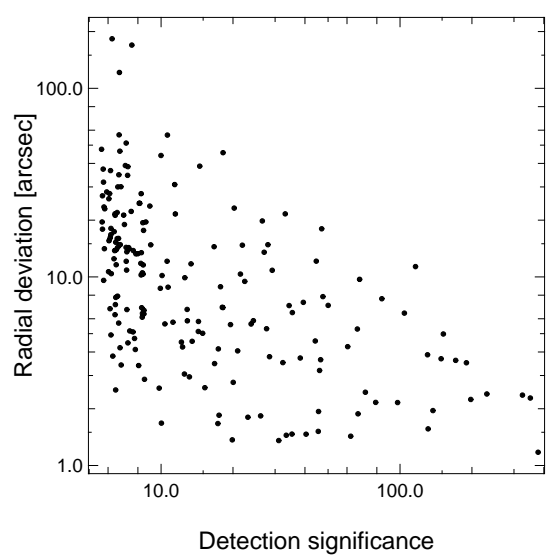

Figure 4: The source position deviation in the mosaic images from the reference catalog plotted against the detection significance. Note that the significance values cannot be compared directly with the ones from the science window detections.

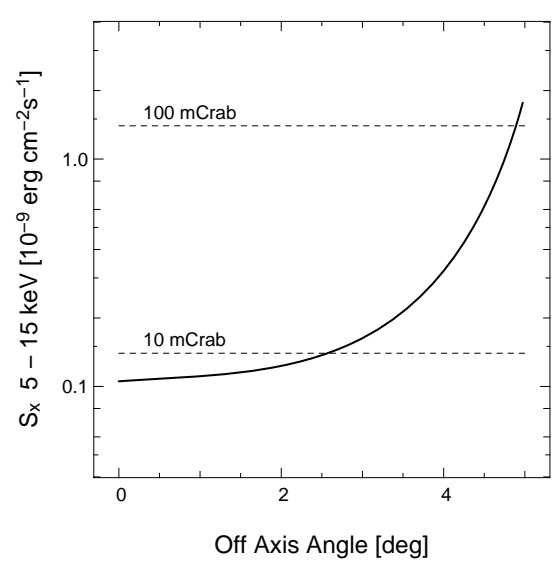

Figure 5: The source detection limit for an observation time of $\sim 2000 \mathrm{~s}$. The dashed lines gives the comparison with the Crab flux. The energy interval $5-15 \mathrm{keV}$ is the range where the signal to noise is most favorable for most sources. 
Table 1: Number of sources in the categories

\begin{tabular}{lccc}
\hline Source category & ScW catalog & Mosaic catalog & Combined catalog \\
\hline \hline Unspecified binaries & 8 & 3 & 10 \\
HMXB & 58 & 58 & 67 \\
LMXB & 79 & 78 & 86 \\
Cataclysmic variables & 3 & 9 & 9 \\
Gamma ray sources & 2 & - & 2 \\
Stellar objects & 2 & 3 & 4 \\
SNR & 4 & 7 & 8 \\
Galaxy clusters & 2 & 1 & 2 \\
AGN & 10 & 30 & 30 \\
Unknown systems & 1 & 1 & 1 \\
\hline
\end{tabular}

\section{Source categories}

Both due to their proximity and the observation pattern (see Fig. 1) the galactic sources dominate the found sources. Table 4 enumerates the various categories according to their classification in the ISDC reference catalog.

\section{The catalog contents}

The catalog itself consists of a file in FITS format that is part of the attachments to this presentation. The first (and only) extension is a binary table with the following columns:

RA_OBJ Right Ascension (J2000) in decimal degrees.

DEC_OBJ Declination (J2000) in decimal degrees.

ERR_RAD The $1 \sigma$ error in radius (degrees).

CLASS Classification according to the HEASARC Object Classification.

DETSIG Combined detections significance.

FLAG Has a value of 1 if only found in the ScW catalog, and of 2 if only found in the mosaic catalog, and of 3 if found in both.

MEANFLUX Mean flux i.e. weighted with the inverse vignetting factor. Given in the energy bands $3-8,8-15$, and $15-25 \mathrm{keV}$. Non-detections are not included.

MEANFLUX_ERR The $1 \sigma$ error of MEANFLUX.

PEAKFLUX The highest flux value found.

PEAKFLUX_ERR The $1 \sigma$ error of PEAKFLUX.

The source position has been given as determined by JEM-X.

\section{Light curves}

Many of the sources in the ScW catalog have been found several times. An accompanying document in PDF format is part of the attachments to this presentation and contains the light curves 
in the three energy bands based on the science window detections scaled to fit the figure height. The time range is from year $2003-2009$ and the 'observability' is shown as well i.e. whether or not it was inside the FOV and how close to the axis. This gives an easy means to check for JEM-X observations of a given source.

\section{Conclusions}

During this work no sources have been found only detected by JEM-X (there is a few examples e.g. Chenevez et al. (2004) of sources first seen by JEM-X). Therefore this catalog becomes a summary of JEM-X observational results with source position information and flux determination including light curves over the 6 year period covered.

\section{Acknowledgements}

I thank Dr. Niels Lund, who has zealously worked on the improvements of the JEM-X image software.

Based on observations with INTEGRAL, an ESA project with instruments and science data centre funded by ESA member states (especially the PI countries: Denmark, France, Germany, Italy, Switzerland, Spain), Czech Republic and Poland, and with the participation of Russia and the USA.

\section{References}

[1] Bird, A.J., Bazzano, A., Bassani, L., et al., 2010, ApJS, 186, 1.

[2] Budtz-Jørgensen, C., Lund, N., Westergaard, N.J. et al., 2006, Proc. SPIE Vol. 6266.

[3] Chenevez et al., 2004, ATEL\#223.

[4] Ebisawa et al., 2003, A\&A 411, 59.

[5] Lund, N. et al., 2003, A\&A, 411, L231.

[6] Westergaard, N.J., Chenevez, J., Lund, N., Budtz-Jørgensen, C., and Brandt, S, 2007, Proc. of the "The 6th Integral Workshop - The obscured Universe", Moscow, ESA SP-622, p. 275. 